\title{
NEW GAMES RELATED TO OLD AND NEW SEQUENCES
}

\author{
A.S. Fraenkel \\ Department of Computer Science and Applied Mathematics, Weizmann Institute of Science, \\ Rehovot 76100, Israel \\ fraenkel@wisdom.weizmann.ac.il, http://www.wisdom.weizmann.ac.il/fraenkel/
}

\begin{abstract}
We define an infinite class of 2-pile subtraction games, where the amount that can be subtracted from both piles simultaneously, is a function $f$ of the size of the piles. Wythoff's game is a special case. For each game, the 2 nd player winning positions are a pair of complementary sequences, some of which are related to well-known sequences, but most are new. The main result is a theorem giving necessary and sufficient conditions on $f$ so that the sequences are 2 nd player winning positions. Sample games are presented, strategy complexity questions are discussed, and possible further studies are indicated.
\end{abstract}

Keywords: 2-pile subtraction games, complexity of games, integer sequences

\section{Introduction}

Dominican International Forwarding is the finest international transportation company, according to its web site (in Spanish) at http://www.dif.com.do . (An optional Google rendition confirms once again that mechanical translation is still in its infancy.) What is the connection of DIF to games?

While pondering this question, let us introduce our first game:

\section{$\mathrm{G}_{1}$ from dif.com}

Given two piles of tokens $(x, y)$ of sizes $x, y$, with $0 \leq x \leq y<\infty$. Two players alternate removing tokens from the piles.

(a) Remove any positive number of tokens from a single pile, possibly the entire pile.

(b) Remove a positive number of tokens from each pile, say $k, \ell$, so that $|k-\ell|$ is not too large with respect to the position $\left(x_{1}, y_{1}\right)$ moved to from $\left(x_{0}, y_{0}\right)$, namely, $|k-\ell|<x_{1}+1\left(x_{1} \leq y_{1}\right)$.

The player making the move after which both piles are empty (a leaf of the game), wins; the opponent loses. Thus, $(11,15) \rightarrow(3,4)$ or to $(2,4)$ are legal 


\begin{tabular}{|l|lllllllllllllllllll|}
\hline$n$ & 0 & 1 & 2 & 3 & 4 & 5 & 6 & 7 & 8 & 9 & 10 & 11 & 12 & 13 & 14 & 15 & 16 \\
\hline$A_{n}$ & 0 & 1 & 3 & 4 & 5 & 7 & 8 & 9 & 10 & 12 & 13 & 14 & 15 & 16 & 18 & 19 & 20 \\
$B_{n}$ & 0 & 2 & 6 & 11 & 17 & 25 & 34 & 44 & 55 & 68 & 82 & 97 & 113 & 130 & 149 & 169 & 190 \\
\hline
\end{tabular}

Table 1. The first few $P$-positions for $\mathbf{G}_{1}$.

moves, but $(11,15) \rightarrow(2,3)$ or to $(0,3)$ are not. The position $(0,0)$ is the only leaf of this and our following games.

For any acyclic combinatorial game without ties, such as $\mathbf{G}_{1}$, a position $u=(x, y)$ is labeled $N$ (Next player win) if the player moving from $u$ can win; otherwise it is a $P$-position (Previous player win). Denote by $\mathcal{P}$ the set of all $P$-positions, by $\mathcal{N}$ the set of all $N$-positions, and by $F(u)$ the set of all (direct) followers or options of $u$. It is easy to see that for any acyclic game,

$$
\begin{gathered}
u \in \mathcal{P} \quad \text { if and only if } \quad F(u) \subseteq \mathcal{N}, \\
u \in \mathcal{N} \quad \text { if and only if } \quad F(u) \cap \mathcal{P} \neq \emptyset .
\end{gathered}
$$

Indeed, player I, beginning from an $N$-position, will move to a $P$-position, which exists by (2), and player II has no choice but to go to an $N$-position, by (1). Since the game is finite and acyclic, player I will eventually win by moving to a leaf, which is clearly a $P$-position.

Let $S \subset \mathbb{Z}_{\geq 0}, S \neq \mathbb{Z}_{\geq 0}$, and $\bar{S}=\mathbb{Z}_{\geq 0} \backslash S$. The minimum excluded value of $S$ is

$$
\operatorname{mex} S=\min \bar{S}=\text { least nonnegative integer not in } S \text {. }
$$

Note that mex of the empty set is 0 .

Table 1 portrays the first few $P$-positions $\left(A_{n}, B_{n}\right)$ of $\mathbf{G}_{1}$. The reader is encouraged to verify that the first few entries of the table are indeed $P$-positions of the game. For a technical reason we put $B_{-1}=-1$. In Section 4 we prove, as a simple corollary to a considerably more general result,

Theorem 1. For $\mathbf{G}_{1}, \mathcal{P}=\cup_{i=0}^{\infty}\left(A_{i}, B_{i}\right)$, where, for all $n \in \mathbb{Z}_{\geq 0}$,

$$
\begin{array}{r}
A_{n}=\operatorname{mex}\left\{A_{i}, B_{i}: 0 \leq i<n\right\}, \\
B_{n}=B_{n-1}+A_{n}+1 .
\end{array}
$$

The game $\mathbf{G}_{1}$ is a special case of the following new family of combinatorial games defined on two piles of finitely many tokens, with two types of moves: a move of type (a), and a more general move of type (b), namely, $|k-\ell|$ depends 


\begin{tabular}{|l|lllllllllllllllll|}
\hline$n$ & 0 & 1 & 2 & 3 & 4 & 5 & 6 & 7 & 8 & 9 & 10 & 11 & 12 & 13 & 14 & 15 & 16 \\
\hline$A_{n}$ & 0 & 1 & 3 & 4 & 5 & 7 & 9 & 11 & 12 & 13 & 15 & 16 & 17 & 19 & 20 & 21 & 23 \\
$B_{n}$ & 0 & 2 & 6 & 8 & 10 & 14 & 18 & 22 & 24 & 26 & 30 & 32 & 34 & 38 & 40 & 42 & 46 \\
\hline
\end{tabular}

Table 2. The first few $P$-positions for $\mathbf{G}_{2}$.

\begin{tabular}{|l|rrrrrrrrrrrrrrrrrr|}
\hline$n$ & 0 & 1 & 2 & 3 & 4 & 5 & 6 & 7 & 8 & 9 & 10 & 11 & 12 & 13 & 14 & 15 & 16 \\
\hline$A_{n}$ & 0 & 1 & 3 & 4 & 5 & 7 & 8 & 9 & 10 & 12 & 13 & 14 & 15 & 16 & 17 & 18 & 19 \\
$B_{n}$ & 0 & 2 & 6 & 11 & 20 & 38 & 71 & 136 & 265 & 523 & 1036 & 2061 & 4110 & 8207 & 16400 & 32785 & 65554 \\
\hline
\end{tabular}

Table 3. The first few $P$-positions for $\mathbf{G}_{3}$.

on the present and next position. Denote the present position by $\left(x_{0}, y_{0}\right)$ and the position moved to by $\left(x_{1}, y_{1}\right)$. We then require,

$$
\left|\left(y_{0}-y_{1}\right)-\left(x_{0}-x_{1}\right)\right|=\left|\left(y_{0}-x_{0}\right)-\left(y_{1}-x_{1}\right)\right|<f\left(x_{1}, y_{1}, x_{0}\right),
$$

where $f$ is a real constraint function depending on $x_{1}, y_{1}, x_{0}$. If also $\left(y_{0}-x_{0}\right) \geq$ $\left(y_{1}-x_{1}\right)$, then the requirement becomes $y_{0}<f\left(x_{1}, y_{1}, x_{0}\right)+y_{1}-x_{1}+x_{0}$. The type (b) move defined for $\mathbf{G}_{1}$ is the special case $f=x_{1}+1$. Here are descriptions of two additional games.

\section{$\mathrm{G}_{2}$ from even.com}

Same as $\mathbf{G}_{1}$, except that in (b), $|k-\ell|<x_{1}+1$ is replaced by $|k-\ell|<$ $x_{0}-x_{1}$.

\section{$\mathrm{G}_{3}$ from dif.com}

Same as $\mathbf{G}_{1}$, except that in (b), $|k-\ell|<x_{1}+1$ is replaced by $|k-\ell|<$ $y_{1}-x_{1}+1$.

The first few $P$-positions for $\mathbf{G}_{2}$ and $\mathbf{G}_{3}$ are listed in Tables 2 and 3 respectively. In Section 4 we also prove,

Theorem 2. For $\mathbf{G}_{2}$ and $\mathbf{G}_{3}, \mathcal{P}=\cup_{i=0}^{\infty}\left(A_{i}, B_{i}\right)$, where, for all $n \in \mathbb{Z}_{\geq 0}$, $A_{n}$ is given by (3), and $B_{n}=2 A_{n}$ for $\mathbf{G}_{2}$; and $B_{0}=0$, and for $n \in \mathbb{Z}_{\geq 1}$, $B_{n}=A_{n}+2^{n}-1$ for $\mathbf{G}_{3}$. 
Each of our games is associated with a pair of complementary sequences $A_{n}$, $B_{n}$. A special case is the well-known (classical) Wythoff (1907) game. See also Berlekamp, Conway, and Guy (1982), Blass and Fraenkel (1990), Connell (1959), Coxeter (1953), Dress (1999), Fraenkel (1982), Fraenkel (1984), Fraenkel and Borosh (1973), Fraenkel and Ozery (1998), Landman (2002), Silber (1976), Silber (1977), and Yaglom and Yaglom (1967). In fact, the classical Wythoff game is the case $f\left(x_{1}, y_{1}, x_{0}\right)=1$, whereas the generalization considered in Fraenkel (1982) is the case $f\left(x_{1}, y_{1}, x_{0}\right)=t$ for any fixed $t \in \mathbb{Z}_{>0}$. Whereas the winning strategy of Wythoff's game is associated with sequences related to algebraic integers of the form $\left(2-t+\sqrt{t^{2}+4}\right) / 2(t=1$ is the golden section), our games give rise to an infinity of sequences, some well-known, but mostly new ones.

In Section 2 we shall see that the pair of sequences of $P$-positions associated with $\mathbf{G}_{1}$ is related to a "self-generating" sequence (Sloane, 1999) of Hofstadter. In Section 3 we indicate how the $P$-positions of $\mathbf{G}_{2}$ are related to another wellknown sequence. The central result appears in Section 4, where a general theorem is formulated and proved, that yields winning strategies for a large class of 2-pile subtraction games. Roughly speaking it states that for every 2-pile subtraction game, if its constraint function $f$ is "positive", "monotone" and "semi-additive", then it has $P$-positions $A_{n}, B_{n}$, where $A_{n}$ satisfies (3), and $B_{n}$ has an explicit form depending on $f$. In a complementary proposition we show that positivity, monotonicity and semi-additivity are also necessary, in the sense that if any one of them is dropped, then there are constraint functions and their associated games $G$, such that the positions claimed to be $P$-positions by the central result, are not $P$-positions for these $G$. Theorems 1 and 2 are then deduced as a simple corollary of the central result. In Section 5 we give a random assortment of sample games with their $P$-positions that can be produced from the central theorem. Questions of complexity and related issues are discussed in Section 6. The epilogue in Section 7 wraps up with some concluding remarks and indications for further study.

\section{The Gödel, Escher, Bach Connection}

On p. 73 of Hofstadter's (1979) famous book the reader is asked to characterize the following sequence:

$$
B_{n \geq 0}^{\prime}=\{1,3,7,12,18,26,35,45,56, \ldots\} .
$$

Answer: the sequence $\{2,4,5,6,8,9,10,11, \ldots\}$ constitutes the set of differences of consecutive terms of $B_{n}^{\prime}$, as well as the complement with respect to $\mathbb{Z}_{>0}$ of $B_{n}^{\prime}$. For our purposes it is convenient to preface 0 to the latter sequence, so we define

$$
A_{n \geq 0}^{\prime}=\{0,2,4,5,6,8,9,10,11, \ldots\},
$$


which is the complement with respect to $\mathbb{Z}_{\geq 0}$ of $B_{n}^{\prime}$. Now $A_{10}^{\prime}=\operatorname{mex}\left\{A_{i}^{\prime}, B_{i}^{\prime}\right.$ : $0 \leq i<10\}=13$, so $B_{10}^{\prime}=56+13=69$. We see that in general, for all $n \in \mathbb{Z}_{\geq 0}$,

$$
A_{n}^{\prime}=\operatorname{mex}\left\{A_{i}^{\prime}, B_{i}^{\prime}: 0 \leq i<n\right\}
$$

which has the form (3), and

$$
B_{-1}^{\prime}=1, \quad B_{n}^{\prime}=B_{n-1}^{\prime}+A_{n}^{\prime},
$$

which is similar to (4). Moreover, the following proposition shows that there is a very close relationship between the $P$-positions of the game $\mathbf{G}_{1}$ and Hofstadter's sequence $B_{n}^{\prime}$, namely, $B_{n}^{\prime}$ exceeds $B_{n}$ by 1 . This can be observed by comparing the bottom row of Table 1 with $B_{n}^{\prime}$.

Proposition 1. $A_{n}^{\prime}=A_{n}+1(n \geq 1), \quad B_{n}^{\prime}=B_{n}+1(n \geq 0)$, where $A_{n}^{\prime}$, $B_{n}^{\prime}$ are given by (6), (7) respectively, and $A_{n}, B_{n}$ by (3), (4) respectively.

Proof. We see that the assertions are true for small $n$. Suppose they hold for all $i \leq n$. Then

$$
A_{n+1}^{\prime}=\operatorname{mex}\left\{A_{i}^{\prime}, B_{i}^{\prime}: 0 \leq i \leq n\right\}=\operatorname{mex}\left\{0, A_{i}+1, B_{i}+1: 0 \leq i \leq n\right\} \text {. }
$$

Put $S_{n}^{\prime}=\left\{0, A_{i}+1, B_{i}+1: 0 \leq i \leq n\right\}, S_{n}=\left\{A_{i}, B_{i}: 0 \leq i \leq n\right\}$. If, say, the integer interval $[0, k]$ is in $S_{n}$ for some $k \in \mathbb{Z}_{>0}$ and $k+1 \notin S_{n}$, then $k+1 \in S_{n}^{\prime}$ and $k+2 \notin S_{n}^{\prime}$. It follows that mex $S_{n}^{\prime}=A_{n+1}+1$. Also, $B_{n+1}^{\prime}=B_{n}^{\prime}+A_{n+1}^{\prime}=B_{n}+1+A_{n+1}+1=B_{n+1}+1$.

Thus the $P$-positions of $G_{1}$ constitute a "translation by 1 " of the Hofstadter sequence, that is, $B_{n+1}-B_{n}=A_{n+1}+1$. So $A_{n}+1$ is the difference (dif) and $A_{n}$ the complement (com) of $B_{n}$ : they are products of dif.com.

\section{Prouhet-Thue-Morse}

It is not hard to see that the sequence $A_{n}(n \geq 1)$ of $\mathbf{G}_{2}$ contains precisely all positive integers whose binary representation ends in even number of zeros. (Because of this, $\mathbf{G}_{2}$ originates from even.com: "www.even.com is the best place to find information and sources for even", it says on its webpage.) The sequence $A_{n}$ is also lexicographically minimal with respect to the property that the parity of number of 1's in the binary expansion alternates. Furthermore, it is lexicographically minimal with respect to the property that the sequence is the double of its complement. If $m$ appears in $A_{n}$, then $2 m$ appears in $B_{n}$. In particular, $B_{n}$ contains precisely all positive integers whose binary representation ends in an odd number of zeros (Carlitz, Scoville, and Hoggatt, 1972). The sequence

$$
\begin{aligned}
C_{n} & =0^{A_{1}-A_{0}} 1^{A_{2}-A_{1}} 0^{A_{3}-A_{2}} \ldots 0^{A_{2 n+1}-A_{2 n}} 1^{A_{2 n+2}-A_{2 n+1}} \ldots \\
& =011010011001011010010 \ldots
\end{aligned}
$$


is the Prouhet-Thue-Morse sequence, which arises in many different areas of mathematics. See the charming paper (Allouche and Shallit, 1999), which also contains $A_{n}$, for many further properties of these sequences.

\section{A Master Theorem}

The three previously described games $\mathbf{G}_{1}, \mathbf{G}_{2}, \mathbf{G}_{3}$, are special cases of an infinite family of games that we now formulate. We shall then provide a general winning strategy for this family of games and prove its validity.

\section{General 2-pile subtraction games}

Given two piles of tokens $(x, y)$ of sizes $x, y$, with $0 \leq x \leq y<\infty$, whose $P$-positions are $\mathcal{P}=\cup_{i=0}^{\infty}\left(A_{i}, B_{i}\right)$. Two players alternate removing tokens from the piles:

(aa) Remove any positive number of tokens from a single pile, possibly the entire pile.

(bb) Remove a positive number of tokens from each pile, say $k, \ell$, so that $|k-\ell|$ is not too large with respect to the position $\left(x_{1}, y_{1}\right)$ moved to from $\left(x_{0}, y_{0}\right)$, namely, $|k-\ell|<f\left(x_{1}, y_{1}, x_{0}\right)$, equivalently:

$$
\left|\left(y_{0}-y_{1}\right)-\left(x_{0}-x_{1}\right)\right|=\left|\left(y_{0}-x_{0}\right)-\left(y_{1}-x_{1}\right)\right|<f\left(x_{1}, y_{1}, x_{0}\right),
$$

where the constraint function $f\left(x_{1}, y_{1}, x_{0}\right)$ is integer-valued and satisfies:

- Positivity:

$$
f\left(x_{1}, y_{1}, x_{0}\right)>0 \forall y_{1} \geq x_{1} \geq 0 \forall x_{0}>x_{1} .
$$

- Monotonicity:

$$
x_{0}^{\prime}<x_{0} \Longrightarrow f\left(x_{1}, y_{1}, x_{0}^{\prime}\right) \leq f\left(x_{1}, y_{1}, x_{0}\right) .
$$

- Semi-additivity (or generalized triangle inequality) on the $P$-positions, namely: for $n>m \geq 0$,

$$
\sum_{i=0}^{m} f\left(A_{n-1-i}, B_{n-1-i}, A_{n-i}\right) \geq f\left(A_{n-m-1}, B_{n-m-1}, A_{n}\right) .
$$

The player making the move after which both piles are empty wins; the opponent loses.

In view of (8), positivity is a natural condition. Without positivity, a move of type (bb) is not even possible. Monotonicity appears to be a minimal requirement to enforce positivity. Semi-additivity is a convenient condition to have, and many functions are semi-additive. Note that $\mathbf{G}_{1}, \mathbf{G}_{2}, \mathbf{G}_{3}$ clearly satisfy 
positivity and monotonicity; $\mathbf{G}_{1}$ and $\mathbf{G}_{3}$, in whose functions $f$ there is no $A_{n}$, are clearly semi-additive; and $\mathbf{G}_{2}$ is semi-additive with equality. (See also the proof of Theorems 1 and 2 at the end of this section.)

Theorem 3. Let $\mathcal{S}=\cup_{i=0}^{\infty}\left(A_{i}, B_{i}\right)$, where, for all $n \in \mathbb{Z}_{\geq 0}, A_{n}$ is given by (3), $B_{0}=0$, and for all $n \in \mathbb{Z}_{>0}$,

$$
B_{n}=f\left(A_{n-1}, B_{n-1}, A_{n}\right)+B_{n-1}-A_{n-1}+A_{n} .
$$

If $f$ is positive, monotone and semi-additive, then $\mathcal{S}$ is the set of P-positions of a general 2-pile subtraction game with constraint function $f$.

Proof. The definition of $A_{n}$ implies directly,

$$
A_{n}>A_{n-1}
$$

for all $n \in \mathbb{Z}_{>0}$. From (9) we have, for all $n \in \mathbb{Z}_{>0}$,

$$
\begin{aligned}
& B_{n}-B_{n-1}=f\left(A_{n-1}, B_{n-1}, A_{n}\right)+A_{n}-A_{n-1}, \\
& B_{n}-A_{n}=f\left(A_{n-1}, B_{n-1}, A_{n}\right)+B_{n-1}-A_{n-1} .
\end{aligned}
$$

Now $f\left(A_{0}, B_{0}, A_{1}\right)>0$ by positivity, so $B_{1}-B_{0} \geq 2$ by (10), (11). Hence we get, by induction on $n$,

$$
B_{n}-B_{m} \geq 2 \text { for all } n>m \geq 0 .
$$

Similarly we get from (12),

$$
B_{n}-A_{n}>B_{m}-A_{m} \geq 0 \text { for all } n>m \geq 0 .
$$

Let $A=\cup_{n=1}^{\infty} A_{n}$ and $B=\cup_{n=1}^{\infty} B_{n}$. Then $A$ and $B$ are complementary sets of integers, i.e., $A \cup B=\mathbb{Z}_{\geq 1}$ (by (3)), and $A \cap B=\emptyset$. Indeed, if $A_{n}=B_{m}$, then $n>m$ implies that $A_{n}$ is the mex of a set containing $B_{m}=A_{n}$, a contradiction to the mex definition; and $1 \leq n \leq m$ is impossible since

$$
\begin{aligned}
B_{m}= & f\left(A_{m-1}, B_{m-1}, A_{m}\right)+B_{m-1}-A_{m-1}+A_{m} \\
\geq & f\left(A_{m-1}, B_{m-1}, A_{n}\right)+B_{n-1}-A_{n-1}+A_{n} \\
& \quad \text { (by (10), (14) and monotonicity) } \\
> & A_{n} \text { (by positivity) } .
\end{aligned}
$$

Since $B_{n}-B_{n-1} \geq 2$ for all $n \geq 1$ by (13), and since $A$ and $B$ are complementary,

$$
A_{n}-A_{n-1} \in\{1,2\}
$$


for all $n \in \mathbb{Z}_{>0}$. Denote by $\mathcal{P}^{\prime}$ the set of all positions $\left(A_{n}, B_{n}\right)$ satisfying (3) and (9), and let $\mathcal{N}^{\prime}=\mathbb{Z}_{\geq 0} \backslash \mathcal{P}^{\prime}$. For showing that $\mathcal{P}^{\prime}=\mathcal{P}$ and $\mathcal{N}^{\prime}=\mathcal{N}$, it evidently suffices to show two things:

I. Every move from any $\left(A_{n}, B_{n}\right) \in \mathcal{P}^{\prime}$ results in a position in the complement $\mathcal{N}^{\prime}$.

II. From every position $(x, y)$ in the complement $\mathcal{N}^{\prime}$, there is a move to some $\left(A_{n}, B_{n}\right) \in \mathcal{P}^{\prime}$.

(It is useful to note that these two conditions are also necessary: (1) implies that all positions reachable in one move from a $P$-position are $N$-positions; whereas (2) shows that at least one $P$-position is reachable in one move from an $N$-position.)

I. A move of type (aa) from $\left(A_{n}, B_{n}\right) \in \mathcal{P}^{\prime}$ has the form $\left(x, B_{n}\right)$ or $\left(A_{n}, y\right)$ $\left(x<A_{n}, y<B_{n}\right)$. Both are in $\mathcal{N}^{\prime}$ since the sequences $A_{n}, B_{n}$ are strictly increasing. Suppose there is a move of type (bb): $\left(A_{n}, B_{n}\right) \rightarrow\left(A_{j}, B_{j}\right) \in \mathcal{P}^{\prime}$. Then $j<n$. Note that

$$
\begin{aligned}
& \left|\left(B_{n}-B_{j}\right)-\left(A_{n}-A_{j}\right)\right| \\
= & \left|\left(B_{n}-A_{n}\right)-\left(B_{j}-A_{j}\right)\right|=\left(B_{n}-A_{n}\right)-\left(B_{j}-A_{j}\right)
\end{aligned}
$$

by (14). By iterating (9) we have,

$$
\begin{aligned}
& \left(B_{n}-A_{n}\right)-\left(B_{j}-A_{j}\right) \\
& =f\left(A_{n-1}, B_{n-1}, A_{n}\right)+\left(B_{n-1}-A_{n-1}\right)-\left(B_{j}-A_{j}\right) \\
& =f\left(A_{n-1}, B_{n-1}, A_{n}\right)+f\left(A_{n-2}, B_{n-2}, A_{n-1}\right) \\
& \quad+\left(B_{n-2}-A_{n-2}\right)-\left(B_{j}-A_{j}\right) \\
& \vdots \\
& =\sum_{i=0}^{n-j-1} f\left(A_{n-i-1}, B_{n-i-1}, A_{n-i}\right) \geq f\left(A_{j}, B_{j}, A_{n}\right),
\end{aligned}
$$

where the inequality follows from semi-additivity. Thus

$$
\left|\left(B_{n}-B_{j}\right)-\left(A_{n}-A_{j}\right)\right| \geq f\left(A_{j}, B_{j}, A_{n}\right),
$$

contradicting condition (bb).

II. Let $(x, y) \in \mathcal{N}^{\prime}(0 \leq x \leq y)$. Since $A$ and $B$ are complementary, every $n \in \mathbb{Z}_{>0}$ appears exactly once in exactly one of $A$ and $B$. Therefore we have either $x=B_{n}$ or else $x=A_{n}$ for some $n \geq 0$.

(i) $x=B_{n}$. Then move $y \rightarrow A_{n}$. This is always possible since if $n=0$, then $y>A_{0}=B_{0}$; whereas $A_{n}<B_{n}$ for $n \geq 1$ by (14). 
(ii) $x=A_{n}$. If $y>B_{n}$, move $y \rightarrow B_{n}$. So suppose that $A_{n} \leq y<B_{n}$. Then $n \geq 1$. For any $m \in\{0, \ldots, n-1\}$ we have by (9) and by monotonicity,

$$
\begin{aligned}
\left(B_{m+1}-A_{m+1}\right)-\left(B_{m}-A_{m}\right) & =f\left(A_{m}, B_{m}, A_{m+1}\right) \\
& \leq f\left(A_{m}, B_{m}, A_{n}\right) .
\end{aligned}
$$

Thus $B_{m}-A_{m}+f\left(A_{m}, B_{m}, A_{n}\right) \geq B_{m+1}-A_{m+1}$. Therefore the intervals $\left[B_{m}-A_{m}, B_{m}-A_{m}+f\left(A_{m}, B_{m}, A_{n}\right)\right)$ (closed on the left, open on the right) cover $\mathbb{Z}_{\geq 0}$ for $m \geq 0$. Hence

$$
y-A_{n} \in\left[B_{m}-A_{m}, B_{m}-A_{m}+f\left(A_{m}, B_{m}, A_{n}\right)\right)
$$

for a smallest $m \in\{0, \ldots, n-1\}$. We then move $(x, y) \rightarrow\left(A_{m}, B_{m}\right)$. This move is legal, since:

- $m<n$. Indeed, $y-A_{n}<B_{n}-A_{n}=f\left(A_{n-1}, B_{n-1}, A_{n}\right)+B_{n-1}-$ $A_{n-1}$. Thus $m \leq n-1$ by (16).

- $y>B_{m}$. By (16), $y-A_{n} \geq B_{m}-A_{m}$. Hence $y-B_{m} \geq A_{n}-A_{m}>0$.

- The move satisfies (bb):

$$
\begin{aligned}
\left|\left(y-B_{m}\right)-\left(x-A_{m}\right)\right| & =\left|\left(y-A_{n}\right)-\left(B_{m}-A_{m}\right)\right| \\
& =\left(y-A_{n}\right)-\left(B_{m}-A_{m}\right)
\end{aligned}
$$

where the last equality follows from (16) and our choice of $m$. We thus have $\left|\left(y-A_{n}\right)-\left(B_{m}-A_{m}\right)\right|=\left(y-A_{n}\right)-\left(B_{m}-A_{m}\right)<$ $f\left(A_{m}, B_{m}, A_{n}\right)$ by (16).

In a sense, Theorem 3 is best possible. This is enunciated below.

Proposition 2. There exist 2-pile subtraction games with constraint functions $f$ which lack precisely one of positivity, monotonicity or semi-additivity, such that $\mathcal{S} \neq \mathcal{P}$, where $\mathcal{S}=\cup_{i=0}^{\infty}\left(A_{i}, B_{i}\right)$, and $A_{i}$ satisfies $(3)\left(i \in \mathbb{Z}_{\geq 0}\right) ; B_{0}=0$, $B_{i}$ satisfies $(9)\left(i \in \mathbb{Z}_{>0}\right)$.

Proof. Consider the function $f\left(x_{1}, y_{1}, x_{0}\right)=\left(x_{0}-x_{1}\right)^{2}$. It is clearly positive and monotone. However, $\left(A_{n}-A_{n-1}\right)^{2}+\left(A_{n-1}-A_{n-2}\right)^{2}<\left(A_{n}-A_{n-2}\right)^{2}$, no matter whether $A_{n}-A_{n-1}=A_{n-1}-A_{n-2}=1$ or otherwise, so $f$ is not semi-additive. From (9) we get, $B_{n}=B_{n-1}+\left(A_{n}-A_{n-1}\right)\left(A_{n}-A_{n-1}+1\right)$, where $A_{n}$ satisfies (3). The first few values of $\left(A_{n}, B_{n}\right)$ are depicted in Table 4. Note that these are not $P$-positions: we can move $\left(A_{n}, B_{n}\right) \rightarrow\left(A_{i}, B_{i}\right)$ in many ways; e.g., $(4,10) \rightarrow(0,0)$ satisfies (bb).

The function $f\left(x_{1}, y_{1}, x_{0}\right)=\left\lfloor\left(x_{1}+1\right) / x_{0}\right\rfloor+1$ is positive. Since

$$
\left(\left\lfloor\frac{A_{n-1}+1}{A_{n}}\right\rfloor+1\right)+\left(\left\lfloor\frac{A_{n-2}+1}{A_{n-1}}\right\rfloor+1\right)>\left\lfloor\frac{A_{n-2}+1}{A_{n}}\right\rfloor+1=1,
$$




\begin{tabular}{|c|ccccccccccccccccc|}
\hline$n$ & 0 & 1 & 2 & 3 & 4 & 5 & 6 & 7 & 8 & 9 & 10 & 11 & 12 & 13 & 14 & 15 & 16 \\
\hline$A_{n}$ & 0 & 1 & 3 & 4 & 5 & 6 & 7 & 9 & 11 & 13 & 15 & 17 & 18 & 19 & 20 & 21 & 23 \\
$B_{n}$ & 0 & 2 & 8 & 10 & 12 & 14 & 16 & 22 & 28 & 34 & 40 & 46 & 48 & 50 & 52 & 54 & 60 \\
\hline
\end{tabular}

Table 4. The first few values of $\mathcal{S}$ for $f=\left(x_{0}-x_{1}\right)^{2}$.

\begin{tabular}{|l|lllllllllllllllllll|}
\hline$n$ & 0 & 1 & 2 & 3 & 4 & 5 & 6 & 7 & 8 & 9 & 10 & 11 & 12 & 13 & 14 & 15 & 16 \\
\hline$A_{n}$ & 0 & 1 & 2 & 4 & 5 & 7 & 8 & 10 & 11 & 13 & 14 & 16 & 17 & 19 & 20 & 22 & 23 \\
$B_{n}$ & 0 & 3 & 6 & 9 & 12 & 15 & 18 & 21 & 24 & 27 & 30 & 33 & 36 & 39 & 42 & 45 & 48 \\
\hline
\end{tabular}

Table 5. The first few values of $\mathcal{S}$ for $f=\left\lfloor\left(x_{1}+1\right) / x_{0}\right\rfloor+1$.

\begin{tabular}{|l|llllllllllllllllll|}
\hline$n$ & 0 & 1 & 2 & 3 & 4 & 5 & 6 & 7 & 8 & 9 & 10 & 11 & 12 & 13 & 14 & 15 & 16 \\
\hline$A_{n}$ & 0 & 1 & 2 & 4 & 5 & 6 & 8 & 9 & 10 & 11 & 14 & 15 & 16 & 17 & 18 & 19 & 20 \\
$B_{n}$ & 0 & 1 & 3 & 7 & 12 & 13 & 21 & 30 & 31 & 42 & 45 & 60 & 61 & 78 & 79 & 98 & 99 \\
\hline
\end{tabular}

Table 6. The first few values of $P$-positions for $f=\left(1+(-1)^{y_{1}+1}\right) x_{1} / 2$.

it is also semi-additive. But it is not monotone. From (9), $B_{n}=B_{n-1}-A_{n-1}+$ $A_{n}+\left\lfloor\left(A_{n-1}+1\right) / A_{n}\right\rfloor+1$. The first few values of $\mathcal{S}=\cup_{n=0}^{\infty}\left(A_{n}, B_{n}\right)$ are shown in Table 5 . The game-position $(4,7) \notin \mathcal{S}$, but it cannot be moved into $\mathcal{S}$. Hence $\mathcal{S} \neq \mathcal{P}$. (Incidentally, note that the sequence $B_{n}$ consists of all nonnegative multiples of 3.)

Lastly, consider $f\left(x_{1}, y_{1}, x_{0}\right)=\left(1+(-1)^{y_{1}+1}\right) x_{1} / 2$. We see easily that $f$ is semi-additive, and it is trivially monotone. But whenever $y_{1}$ is even, $f$ is not positive. We have, $B_{n}=A_{n}+B_{n-1}-\left(1+(-1)^{B_{n-1}}\right) A_{n-1} / 2$. Table 6 shows the first few $\mathcal{S}$-positions. These are not $P$-positions: The position $(10,29) \notin \mathcal{S}$, cannot be moved into any position in $\mathcal{S}$.

Proof of Theorems 1 and 2. The function $f\left(x_{1}, y_{1}, x_{0}\right)=x_{1}+1$ is clearly positive. Monotonicity is satisfied trivially. It is also clear that $f$ is semiadditive. The function $f\left(x_{1}, y_{1}, x_{0}\right)=x_{0}-x_{1}$ is positive, since $x_{0}>x_{1}$. It's also monotone. Since $\left(A_{n+1}-A_{n}\right)+\left(A_{n}-A_{n-1}\right)=A_{n+1}-A_{n-1}$, we see that $f$ is semi-additive. Finally, the function $f\left(x_{1}, y_{1}, x_{0}\right)=y_{1}-x_{1}+1$ 


\begin{tabular}{|r|rrrrrrrrrrrrrrrrrr|}
\hline$n$ & 0 & 1 & 2 & 3 & 4 & 5 & 6 & 7 & 8 & 9 & 10 & 11 & 12 & 13 & 14 & 15 & 16 \\
\hline$A_{n}$ & 0 & 1 & 3 & 4 & 5 & 6 & 8 & 9 & 10 & 11 & 13 & 14 & 15 & 16 & 17 & 19 & 20 \\
$B_{n}$ & 0 & 2 & 7 & 12 & 18 & 25 & 35 & 45 & 56 & 68 & 83 & 98 & 114 & 131 & 149 & 170 & 191 \\
\hline
\end{tabular}

Table 7. The first few values of $\mathcal{S}$ for $f=x_{1}-\left\lfloor\left(x_{1}+1\right) / x_{0}\right\rfloor+2$.

\begin{tabular}{|c|ccccccccccccccccc|}
\hline$n$ & 0 & 1 & 2 & 3 & 4 & 5 & 6 & 7 & 8 & 9 & 10 & 11 & 12 & 13 & 14 & 15 & 16 \\
\hline$A_{n}$ & 0 & 1 & 2 & 3 & 5 & 6 & 7 & 9 & 10 & 11 & 13 & 14 & 15 & 16 & 17 & 19 & 20 \\
$B_{n}$ & 0 & 4 & 8 & 12 & 18 & 22 & 26 & 32 & 36 & 40 & 46 & 50 & 54 & 58 & 62 & 68 & 72 \\
\hline
\end{tabular}

Table 8. The first few values of $\mathcal{S}$ for $f=x_{0}-x_{1}+2$.

is positive for all $x_{1} \leq y_{1}$ and is trivially monotone. It is also semi-additive. Thus by Theorem 3 we have for $\mathbf{G}_{1}, B_{n}=A_{n-1}+1+B_{n-1}-A_{n-1}+A_{n}=$ $B_{n-1}+A_{n}+1$, as stated in Theorem 1. For $\mathbf{G}_{2}$, (9) implies, $B_{n}=A_{n}-$ $A_{n-1}+B_{n-1}-A_{n-1}+A_{n}=2 A_{n}-2 A_{n-1}+B_{n-1}=2 A_{n}$, where the last equality follows by induction on $n$. For $\mathrm{G}_{3}, B_{n}=B_{n-1}-A_{n-1}+1+B_{n-1}-$ $A_{n-1}+A_{n}=2\left(B_{n-1}-A_{n-1}\right)+A_{n}+1=A_{n}+2^{n}-1$. Again the last equality follows by induction.

\section{Further Sample Games}

For the examples below, we leave it to the reader to verify positivity, monotonicity and semi-additivity of $f$. Some of these examples are elaborated on in the next two sections.

Example 1. $f\left(x_{1}, y_{1}, x_{0}\right)=x_{1}-\left\lfloor\left(x_{1}+1\right) / x_{0}\right\rfloor+2$. Then $B_{n}=B_{n-1}+$ $A_{n}-\left\lfloor\left(A_{n-1}+1\right) / A_{n}\right\rfloor+2$. The first few $P$-positions are depicted in Table 7 .

Example 2. $f\left(x_{1}, y_{1}, x_{0}\right)=x_{0}-x_{1}+2$. Then $B_{n}=B_{n-1}+2\left(A_{n}-A_{n-1}+1\right)$. See Table 8 for the first few $P$-positions.

Example 3. $f\left(x_{1}, y_{1}, x_{0}\right)=(-1)^{y_{1}}-(-1)^{x_{1}}+3$. Then $B_{n}=B_{n-1}-A_{n-1}+$ $A_{n}+(-1)^{B_{n-1}}-(-1)^{A_{n-1}}+3$. See Table 9 for the first few $P$-positions.

Example 4. $f\left(x_{1}, y_{1}, x_{0}\right)=x_{1}\left(1+(-1)^{x_{1}}\right)+1$. This leads to $B_{n}=B_{n-1}+$ $(-1)^{A_{n-1}} A_{n-1}+A_{n}+1$. Table 10 exhibits the first few $P$-positions. 


\begin{tabular}{|c|ccccccccccccccccc|}
\hline$n$ & 0 & 1 & 2 & 3 & 4 & 5 & 6 & 7 & 8 & 9 & 10 & 11 & 12 & 13 & 14 & 15 & 16 \\
\hline$A_{n}$ & 0 & 1 & 2 & 3 & 5 & 6 & 7 & 8 & 9 & 11 & 12 & 13 & 15 & 16 & 17 & 18 & 19 \\
$B_{n}$ & 0 & 4 & 10 & 14 & 21 & 25 & 27 & 31 & 33 & 38 & 44 & 48 & 55 & 59 & 61 & 65 & 67 \\
\hline
\end{tabular}

Table 9. The first few values of $\mathcal{S}$ for $f=(-1)^{y_{1}}-(-1)^{x_{1}}+3$.

\begin{tabular}{|l|lllllllllllllllllll|}
\hline$n$ & 0 & 1 & 2 & 3 & 4 & 5 & 6 & 7 & 8 & 9 & 10 & 11 & 12 & 13 & 14 & 15 & 16 \\
\hline$A_{n}$ & 0 & 1 & 3 & 4 & 6 & 8 & 9 & 10 & 11 & 12 & 13 & 14 & 15 & 16 & 17 & 19 & 20 \\
$B_{n}$ & 0 & 2 & 5 & 7 & 18 & 33 & 42 & 44 & 66 & 68 & 94 & 96 & 136 & 138 & 172 & 175 & 177 \\
\hline
\end{tabular}

Table 10. The first few values of $\mathcal{S}$ for $f=x_{1}\left(1+(-1)^{x_{1}}\right)+1$.

\section{Computational Complexity Issues}

What is the computational complexity of computing the winning strategy for our games? Given a position $(x, y)$ with $0 \leq x \leq y<\infty$, the statement of Theorem 3 enables us to compute the table of $P$-positions. It suffices to compute it up to the smallest $n=n_{0}$ such that $A_{n_{0}} \geq x$, and thus determine whether $(x, y) \in \mathcal{P}$ or in $\mathcal{N}$. The proof of Theorem 3 then enables us, if $(x, y) \in \mathcal{N}$, to make a winning move to a position in $\mathcal{P}$. The latter part of the strategy, that of making a winning move, is clearly polynomial. The first part, determining whether or not $(x, y) \in \mathcal{P}$ is linear in $x$, since $A_{n_{0}} \leq 2 x$ by (15).

Our games, however, are succinct, i.e., the input size is $\Omega(\log x)$ rather than $\Omega(x)$ (assuming that $y$ is bounded by a polynomial in $x$ ). Thus their complexity is not obvious a priori. Even if the $B_{n}$-sequence grows exponentially, polynomiality of the strategy does not necessarily follow. For example, I do not know whether the sequence $B_{n}$ of $\mathbf{G}_{3}$ can be computed polynomially.

Special sequences are known to be computable polynomially. For example, consider the numeration system with bases defined by the recurrence $u_{n}=$ $(s+t-1) u_{n-1}+s u_{n-2}(n \geq 1)$, where $s, t \in \mathbb{Z}_{>0}$, with initial conditions $u_{-1}=1 / s, u_{0}=1$. It follows from Fraenkel (1985) that every positive integer $N$ has a unique representation of the form $N=\sum_{i \geq 0} d_{i} u_{i}$, with digits $d_{i} \in\{0, \ldots, s+t-1\}$, such that $d_{i+1}=s+t-1 \Longrightarrow d_{i}<s$ for all $i \in \mathbb{Z}_{\geq 0}$. The representation of the first few entries for the special case $s=2, t=2$, is depicted in Table 10.

If we compare Table 11 with Table 8, we might note the following two properties: 


\begin{tabular}{|c|c|c|c|c|c|c|c|c|}
\hline 50 & 14 & 4 & 1 & $n$ & 14 & 4 & 1 & $n$ \\
\hline & 2 & 0 & 3 & 31 & & & 1 & 1 \\
\hline & 2 & 1 & 0 & 32 & & & 2 & 2 \\
\hline & 2 & 1 & 1 & 33 & & & 3 & 3 \\
\hline & 2 & 1 & 2 & 34 & & 1 & 0 & 4 \\
\hline & 2 & 1 & 3 & 35 & & 1 & 1 & 5 \\
\hline & 2 & 2 & 0 & 36 & & 1 & 2 & 6 \\
\hline & 2 & 2 & 1 & 37 & & 1 & 3 & 7 \\
\hline & 2 & 2 & 2 & 38 & & 2 & 0 & 8 \\
\hline & 2 & 2 & 3 & 39 & & 2 & 1 & 9 \\
\hline & 2 & 3 & 0 & 40 & & 2 & 2 & 10 \\
\hline & 2 & 3 & 1 & 41 & & 2 & 3 & 11 \\
\hline & 3 & 0 & 0 & 42 & & 3 & 0 & 12 \\
\hline & 3 & 0 & 1 & 43 & & 3 & 1 & 13 \\
\hline & 3 & 0 & 2 & 44 & 1 & 0 & 0 & 14 \\
\hline & 3 & 0 & 3 & 45 & 1 & 0 & 1 & 15 \\
\hline & 3 & 1 & 0 & 46 & 1 & 0 & 2 & 16 \\
\hline & 3 & 1 & 1 & 47 & 1 & 0 & 3 & 17 \\
\hline & 3 & 1 & 2 & 48 & 1 & 1 & 0 & 18 \\
\hline & 3 & 1 & 3 & 49 & 1 & 1 & 1 & 19 \\
\hline 1 & 0 & 0 & 0 & 50 & 1 & 1 & 2 & 20 \\
\hline 1 & 0 & 0 & 1 & 51 & 1 & 1 & 3 & 21 \\
\hline 1 & 0 & 0 & 2 & 52 & 1 & 2 & 0 & 22 \\
\hline 1 & 0 & 0 & 3 & 53 & 1 & 2 & 1 & 23 \\
\hline 1 & 0 & 1 & 0 & 54 & 1 & 2 & 2 & 24 \\
\hline 1 & 0 & 1 & 1 & 55 & 1 & 2 & 3 & 25 \\
\hline 1 & 0 & 1 & 2 & 56 & 1 & 3 & 0 & 26 \\
\hline 1 & 0 & 1 & 3 & 57 & 1 & 3 & 1 & 27 \\
\hline 1 & 0 & 2 & 0 & 58 & 2 & 0 & 0 & 28 \\
\hline 1 & 0 & 2 & 1 & 59 & 2 & 0 & 1 & 29 \\
\hline 1 & 0 & 2 & 2 & 60 & 2 & 0 & 2 & 30 \\
\hline
\end{tabular}

Table 11. Representation of the first few integers in a special numeration system.

- All the $A_{n}$ have representations ending in an even number of 0 s, and all the $B_{n}$ have representations ending in an odd number of $0 \mathrm{~s}$.

- For every $\left(A_{n}, B_{n}\right) \in \mathcal{P}$, the representation of $B_{n}$ is the "left shift" of the representation of $A_{n}$.

Thus $(1,4)$ of Table 11 has representation $(1,10)$, and $(6,22)$ has representation $(12,120): 10$ is the "left shift" of 1,120 the left shift of 12 .

These properties hold, in fact, in general for Example 2, which is a special case of another family of sequences and games analyzed in Fraenkel (1998). They enable one to win in polynomial time for that family.

However, we do not even know whether there are NP-hard sequences. A case in point is the infinite family of octal games (Guy and Smith, 1956; Berlekamp, 
Conway, and Guy, 1982, ch. 4), even for the subfamily where there are only finitely many nonzero octal digits. Some octal games have been shown to have polynomial strategies (see, e.g., Gangolli and Plambeck, 1989) but the complexity of most is unknown.

We mention very briefly other relevant complexities. They include Kolmogorov complexity, subword complexity, palindrome complexity, and, we might add, squares complexity. The subword complexity $c(n)$ of a sequence $S$ is the number of distinct words of length $n$ occurring in $S$. In Allouche et al. (2003), this notion is attributed to Ehrenfeucht, Lee, and Rozenberg (1975). Surveys can be found in Allouche (1994), Ferenczi (1999), Ferenczi and Kása (1999). The palindrome complexity $p(n)$ of $S$ is the number of distinct palindromes of length $n$ in $S$. See, e.g., Damanik and Zare (2000), and Allouche and Shallit (2003). Define the squares complexity $s(n)$ of $S$ as the number of distinct squares of length $n$ in $S$. Thus the result of Fraenkel and Simpson (1995) implies that there are binary sequences for which $s(2)=2, s(4)=1$, $s(2 k)=0$ for all $k>2$. There is also the notion of program complexity (Daley, $1973,1974,1975)$ concerning the complexity of computing a sequence, which is related to Kolmogorov (1968) complexity.

\section{Epilogue}

We have defined an infinite class of 2-pile subtraction games with two types of moves: (aaa) remove any positive number from a single pile; (bbb) remove $k>0$ from one pile, $\ell>0$ from the other. This move is restricted by the requirement $|k-\ell|<f$, where $f$ is a positive real-valued function. We have shown that a pair $A_{n}, B_{n}$ of judiciously chosen complementary sequences constitutes the set of $P$-positions if and only if $f$ is monotone and semi-additive.

As we have pointed out, the generalized Wythoff game (Fraenkel, 1982) is a special case of the family of games considered here. It has the property that a polynomial strategy can be given by using a natural numeration system, and noting that the $A_{n}$ members are characterized by ending in an even number of $0 \mathrm{~s}$ in that representation, and the $B_{n}$ being their left shifts. A similar situation exists for $\mathbf{G}_{2}$, but with the standard binary representation as numeration system. With the game in Example 2, an essentially different numeration system (see Fraenkel, 1998) can be associated to the same effect.

\section{Further studies}

1. With which games can we associate an appropriate numeration system so as to establish a polynomial strategy?

2. Extend the games in a natural way to handle more than two piles. For Wythoff's game, I have a conjecture (see Guy and Nowakowski, 2002, Problem 53; Fraenkel, 2003, Section 5). 
3. Compute the Sprague-Grundy function $g$ for the games, which will enable to play sums of games. For Wythoff's game this is an as yet unsolved problem, though eventual additive periodicity has been proved (Dress, Flammenkamp, and Pink, 1999; Landman, 2002).

4. Compute a strategy for the games when played in misère version, i.e., the player making the last move loses. This is easy for Wythoff's game (see Berlekamp, Conway, and Guy, 1982, ch. 13).

5. We have already mentioned the question of the polynomiality of the strategy. Is there a 2-pile subtraction game that is Pspace-complete?

6. Computation of complexities of $P$-positions sequences, such as Kolmogorov-, program-, subword-, palindrome-, squares-complexities. For the $A_{n}$ sequence of Example 2, the subword complexity was computed in Fraenkel, Seeman, and Simpson (2001).

7. Make an about-face: begin with pairs of known complementary sequences, and design matching 2-pile subtraction games.

\section{References}

J-P. Allouche, Sur la complexitè des suites infinies, Bull. Belg. Math. Soc. 1 (1994) 133-143.

J-P. Allouche, M. Baake, J. Cassaigne, and D. Damanik, Palindrome complexity, Theoret. Comput. Sci. 292 (2003) 9-31.

J-P. Allouche and J. Shallit, The ubiquitous Prouhet-Thue-Morse sequence, in: Sequences and their applications (Singapore, 1998), 1-16, Springer Ser. Discrete Math. Theor. Comput. Sci., Springer, London, 1999.

E.R. Berlekamp, J.H. Conway, and R.K. Guy, Winning Ways for your Mathematical Plays, Vol. I \& II, Academic Press, London, 1982.

U. Blass and A.S. Fraenkel [1990], The Sprague-Grundy function for Wythoff's game, Theoret. Comput. Sci. (Math Games) 75 (1990) 311-333.

L. Carlitz, R. Scoville and V.E. Hoggatt Jr., Representations for a special sequence, Fibonacci Quart. 10 (1972) 499-518; 550.

I.G. Connell, A generalization of Wythoff's game, Canad. Math. Bull. 2 (1959) 181-190.

H.S.M. Coxeter, The golden section, phyllotaxis and Wythoff's game, Scripta Math 19 (1953) 135-143.

R. P. Daley, Minimal-program complexity of sequences with restricted resources, Information and Control 23 (1973) 301-312.

R. P. Daley, The extent and density of sequences within the minimal-program complexity hierarchies, J. Comput. System Sci. 9 (1974) 151-163.

R. P. Daley, Minimal-program complexity of pseudo-recursive and pseudo-random sequences, Math. Systems Theory 9 (1975) 83-94.

D. Damanik and D. Zare, Palindrome complexity bounds for primitive substitution sequences, Discrete Math. 222 (2000) 259-267.

A. Dress, A. Flammenkamp and N. Pink, Additive periodicity of the Sprague-Grundy function of certain Nim games, Adv. in Appl. Math. 22 (1999) 249-270.

A. Ehrenfeucht. K. P. Lee, G. Rozenberg, Subword complexities of various classes of deterministic developmental languages without interaction, Theoret. Comput. Sci. 1 (1975) 59-75.

S. Ferenczi, Complexity of sequences and dynamical systems, Discrete Math. 206 (1999) 145154. 
S. Ferenczi and Z. Kása, Complexity for finite finite factors of infinite sequences, Theoret. Comput. Sci. 218 (1999) 177-195.

A.S. Fraenkel, How to beat your Wythoff games' opponent on three fronts, Amer. Math. Monthly 89 (1982) 353-361.

A.S. Fraenkel, Wythoff games, continued fractions, cedar trees and Fibonacci searches, Theoret. Comput. Sci. 29 (1984) 49-73.

A.S. Fraenkel, Systems of numeration, Amer. Math. Monthly 92 (1985) 105-114.

A.S. Fraenkel, Heap games, numeration systems and sequences, Annals of Combinatorics 2 (1998) 197-210.

A.S. Fraenkel, Complexity, appeal and challenges of combinatorial games, Theoret. Comp. Sci. Proc. of Dagstuhl Seminar "Algorithmic Combinatorial Game Theory", Feb. 2002; to appear.

A.S. Fraenkel and I. Borosh, A generalization of Wythoff's game, J. Combin. Theory (Ser. A) 15 (1973) 175-191.

A.S. Fraenkel and M. Ozery, Adjoining to Wythoff's game its $P$-positions as moves, Theoret. Comput. Sci. 205 (1998) 283-296.

A.S. Fraenkel, T. Seeman and J. Simpson, The subword complexity of a two-parameter family of sequences, Electronic J. Combinatorics 8(2) (2001) R10, 19pp. http://www.combinatorics.org/

A.S. Fraenkel and R.J. Simpson, How many squares must a binary sequence contain? Electronic J. Combinatorics 2 (1995) R2, 9pp. http://www.combinatorics.org/

A. Gangolli and T. Plambeck, A note on periodicity in some octal games, Internat. J. Game Theory 18 (1989) 311-320.

R.K. Guy and R.J. Nowakowski, Unsolved problems in combinatorial games, in: More Games of No Chance, Proc. MSRI Workshop on Combinatorial Games, July, 2000, Berkeley, CA, MSRI Publ. (R.J. Nowakowski, ed.), Vol. 42, Cambridge University Press, Cambridge, pp. 457-473, 2002.

R.K. Guy and C.A.B. Smith [1956], The G-values of various games, Proc. Camb. Phil. Soc. 52 (1956) 514-526.

D.R. Hofstadter, Gödel, Escher, Bach: An Eternal Golden Braid, Basic Books, New York, 1979.

A. N. Kolmogorov, Three approaches to the quantitative definition of information, Internat. $J$. Comput. Math. 2 (1968) 157-168. (An English translation of the original Russian article in Problemy Peredači Informacii 1 (1965),vyp. 1, 3-11.)

H. Landman, A simple FSM-based proof of the additive periodicity of the Sprague-Grundy function of Wythoff's game, in: More Games of No Chance, Proc. MSRI Workshop on Combinatorial Games, July, 2000, Berkeley, CA, MSRI Publ. (R. J. Nowakowski, ed.), Vol. 42, Cambridge University Press, Cambridge, pp. 383-386, 2002.

R. Silber, A Fibonacci property of Wythoff pairs, Fibonacci Quart. 14 (1976) 380-384.

R. Silber, Wythoff's Nim and Fibonacci representations, Fibonacci Quart. 15 (1977) 85-88.

N.J.A. Sloane, My favorite integer sequences, Sequences and their applications (Singapore, 1998), Springer Ser. Discrete Math. Theor. Comput. Sci., Springer, London, pp. 103-130, 1999.

W.A. Wythoff, A modification of the game of Nim, Nieuw Arch. Wisk. 7 (1907) 199-202.

A.M. Yaglom and I.M. Yaglom, Challenging Mathematical Problems with Elementary Solutions, Vol. II, Holden-Day, San Francisco, translated by J. McCawley, Jr., revised and edited by B. Gordon, 1967. 